EUROPEAN JOURNAL OF PURE AND APPLIED MATHEMATICS

Vol. 13, No. 5, 2020, 1057-1071

ISSN 1307-5543 - www.ejpam.com

Published by New York Business Global

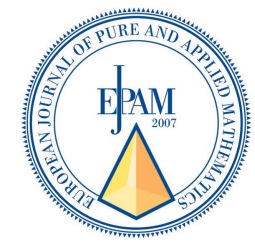

Special Issue Dedicated to

Professor Hari M. Srivastava

On the Occasion of his 80th Birthday

\title{
Transmission and Reciprocal Transmission Based Topological Co-Indices of Graphs
}

\author{
Harishchandra S. Ramane ${ }^{1}$, Saroja Y. Talwar ${ }^{1}$, Ismail Naci Cangul ${ }^{2, *}$ \\ 1 Department of Mathematics, Karnatak University, 580003 Dharwad, India \\ 2 Department of Mathematics, Bursa Uludag University, 16059 Bursa, Turkey
}

\begin{abstract}
The transmission of a vertex $u$ in a connected graph $G$ is defined as the sum of the distances between $u$ and all other vertices of a graph $G$. The reciprocal transmission of a vertex $u$ in a connected graph $G$ is defined as the sum of the reciprocal of distances between $u$ and all other vertices of a graph $G$. In this paper, we introduce and study new topological co-indices based on the transmission and reciprocal transmission of a vertex, such as transmission and reciprocal transmission sum-connectivity co-indices, transmission and reciprocal transmission atom bond connectivity co-indices, transmission and reciprocal transmission geometric-arithmetic coindices, transmission and reciprocal transmission augmented Zagreb co-indices, and transmission and reciprocal transmission arithmetic-geometric co-indices. Further we obtain general formulae for some graphs.
\end{abstract}

2020 Mathematics Subject Classifications: 05C07, 05C30, 05C69

Key Words and Phrases: Transmission of a vertex, reciprocal transmission of a vertex, topological index, graph distance

\section{Introduction}

Topological indices are proved to be very useful in chemistry, biochemistry and nanotechnology in isomer discrimination, structure-property relationship, structure-activity relationship and pharmaceutical drug design. According to the International Academy of

${ }^{*}$ Corresponding author.

DOI: https://doi.org/10.29020/nybg.ejpam.v13i5.3724

Email addresses: cangul@uludag.edu.tr (I. N. Cangul)

hsramane@yahoo.com (H. S. Ramane), sarojaytalwar@gmail.com (S. Y. Talwar) 
Mathematical Chemistry, to identify whether any topological index is useful for prediction of chemical properties, the correlation between the values of that topological index for different octane isomers and parameter values related to certain physicochemical property of them should be considered. Generally octane isomers are convenient for such studies, because the number of the structural isomers of octane is large enough to make the statistical conclusion reliable. Furtula and Gutman [5] showed that for octane isomers both $M_{1}$ and $F$ yield correlation coefficient greater than 0.95 in case of entropy and acentric factor. They also improved the predictive ability of these index by considering a simple linear model in the form $\left(M_{1}+\lambda F\right)$, where $\lambda$ varies from -20 to 20 .

For graph theoretical parameters, we refer the book [10]. Let $G$ be a graph having $n$ vertices and $m$ edges. Let $V(G)$ be the vertex set and $E(G)$ be the edge set of $G$. The edge $e$ joining the vertices $u$ and $v$ is denoted by $e=u v$. $e$ is said to be incident to $u$ and $v$ and $u$ and $v$ are called adjacent. The degree of a vertex $u$ is the number of edges incident to it and is denoted by $d(u)$. The distance between the vertices $u$ and $v$ is the length of the shortest path joining $u$ and $v$ and is denoted by $d(u, v)$. The diameter of $G$ is the maximum distance between all pair of vertices of $G$ and is denoted by $\operatorname{diam}(G)$.

A topological index is a numerical invariant of a given graph [13]. Particular topological indices include the Zagreb indices, ABC index, GA index, Balaban index, Harary index, molecular topological index and Wiener index. Unless otherwise stated, hydrogen atoms are usually ignored in the computation of such indices as organic chemists usually do when they write a benzene ring as a hexagon.

In the literature, several degree based topological indices have been introduced and studied [6]. The most studied degree based topological indices are the family of Zagreb indices, $[1-4,7,12,17,18,20-24,26-28]$. The first and second Zagreb indices of a graph $G$ are defined by

$$
M_{1}(G)=\sum_{u v \in E(G)}[d(u)+d(v)] \text { and } M_{2}(G)=\sum_{u v \in E(G)} d(u) d(v),
$$

see $[8]$.

The transmission (or status) of a vertex $u \in V(G),[9,16]$, denoted by $\sigma(u)$, is defined by

$$
\sigma(u)=\sum_{v \in V(G)} d(u, v)
$$

The reciprocal transmission (or reciprocal status) of a vertex $u \in V(G)$, denoted by $r s(u)$, is defined by

$$
r s(u)=\sum_{v \in V(G)} \frac{1}{d(u, v)} .
$$


The oldest transmission based topological index is the Wiener index [25] defined by

$$
W(G)=\sum_{\{u, v\} \subseteq V(G)} d(u, v)=\frac{1}{2} \sum_{u \in V(G)} \sigma(u) .
$$

The Wiener index is also called as gross status or total status, [9].

The transmission sum-connectivity index of a graph $G$, [19], denoted by $T_{S C}(G)$, is defined by

$$
T_{S C}(G)=\sum_{u v \in E(G)} \frac{1}{\sqrt{\sigma(u)+\sigma(v)}}
$$

The transmission geometric-arithmetic index of a graph $G$, [11], denoted by $T_{G A}(G)$, is defined by

$$
T_{G A}(G)=\sum_{u v \in E(G)} \frac{2 \sqrt{\sigma(u) \sigma(v)}}{\sigma(u)+\sigma(v)} .
$$

The transmission arithmetic-geometric index of a graph $G$, [15], denoted by $T_{A G}(G)$, is defined by

$$
T_{A G}(G)=\sum_{u v \in E(G)} \frac{\sigma(u)+\sigma(v)}{2 \sqrt{\sigma(u) \sigma(v)}} .
$$

The transmission atom-bond connectivity index of a graph $G$, [15], denoted by $T_{A B C}(G)$, is defined by

$$
T_{A B C}(G)=\sum_{u v \in E(G)} \sqrt{\frac{\sigma(u)+\sigma(v)-2}{\sigma(u) \sigma(v)}} .
$$

The transmission augmented Zagreb index of a graph $G$, [15], denoted by $T_{A Z}(G)$, is defined by

$$
T_{A Z}(G)=\sum_{u v \in E(G)}\left[\frac{\sigma(u) \sigma(v)}{\sigma(u)+\sigma(v)-2}\right]^{3} .
$$

The reciprocal transmission arithmetic-geometric index of a graph $G$, [14], is denoted by $R T_{A G}(G)$ and it is defined by

$$
R T_{A G}(G)=\sum_{u v \in E(G)} \frac{r s(u)+r s(v)}{2 \sqrt{r s(u) r s(v)}} .
$$

The reciprocal transmission geometric-arithmetic index of a graph $G$, [14], is denoted by $R T_{G A}(G)$ and it is defined by

$$
R T_{G A}(G)=\sum_{u v \in E(G)} \frac{2 \sqrt{r s(u) r s(v)}}{r s(u)+r s(v)} .
$$


The reciprocal transmission sum-connectivity index of a graph $G$, [14], is denoted by $R T_{S C}(G)$ and it is defined by

$$
R T_{S C}(G)=\sum_{u v \in E(G)} \frac{1}{\sqrt{r s(u)+r s(v)}} .
$$

The reciprocal transmission atom-bond connectivity index of a graph $G$, [14], is denoted by $R T_{A B C}(G)$ and it is defined by

$$
R T_{A B C}(G)=\sum_{u v \in E(G)} \sqrt{\frac{r s(u)+r s(v)-2}{r s(u) r s(v)}} .
$$

The reciprocal transmission augmented Zagreb index of a graph $G$, [14], is denoted by $R T_{A Z}(G)$ and it is defined by

$$
R T_{A Z}(G)=\sum_{u v \in E(G)}\left[\frac{r s(u) r s(v)}{r s(u)+r s(v)-2}\right]^{3} .
$$

In the next section, we obtain bounds for the other transmission and reciprocal transmissionbased topological co-indices.

Now we define the following transmission and reciprocal transmission based topological co-indices of graphs.

The transmission sum-connectivity co-index of a graph $G$, denoted by $\bar{T}_{S C}(G)$, is defined by

$$
\bar{T}_{S C}(G)=\sum_{u v \notin E(G)} \frac{1}{\sqrt{\sigma(u)+\sigma(v)}} .
$$

The transmission geometric-arithmetic co-index of a graph $G$, denoted by $\bar{T}_{G A}(G)$, is defined by

$$
\bar{T}_{G A}(G)=\sum_{u v \notin E(G)} \frac{2 \sqrt{\sigma(u) \sigma(v)}}{\sigma(u)+\sigma(v)} .
$$

The transmission arithmetic-geometric co-index of a graph $G$, denoted by $\bar{T}_{A G}(G)$, is defined by

$$
\bar{T}_{A G}(G)=\sum_{u v \notin E(G)} \frac{\sigma(u)+\sigma(v)}{2 \sqrt{\sigma(u) \sigma(v)}} .
$$

The transmission atom-bond connectivity co-index of a graph $G$, denoted by $\bar{T}_{A B C}(G)$, is defined by

$$
\bar{T}_{A B C}(G)=\sum_{u v \notin E(G)} \sqrt{\frac{\sigma(u)+\sigma(v)-2}{\sigma(u) \sigma(v)}} .
$$


The transmission augmented Zagreb co-index of a graph $G$, denoted by $\bar{T}_{A Z}(G)$, is defined by

$$
\bar{T}_{A Z}(G)=\sum_{u v \notin E(G)}\left[\frac{\sigma(u) \sigma(v)}{\sigma(u)+\sigma(v)-2}\right]^{3} .
$$

The reciprocal transmission arithmetic-geometric co-index of a graph $G$ is denoted by $\overline{R T}_{A G}(G)$ and it is defined by

$$
\overline{R T}_{A G}(G)=\sum_{u v \notin E(G)} \frac{r s(u)+r s(v)}{2 \sqrt{r s(u) r s(v)}} .
$$

The reciprocal transmission geometric-arithmetic co-index of a graph $G$ is denoted by $\overline{R T}_{G A}(G)$ and it is defined by

$$
\overline{R T}_{G A}(G)=\sum_{u v \notin E(G)} \frac{2 \sqrt{r s(u) r s(v)}}{r s(u)+r s(v)} .
$$

The reciprocal transmission sum-connectivity co-index of a graph $G$ is denoted by $\overline{R T}_{S C}(G)$ and it is defined by

$$
\overline{R T}_{S C}(G)=\sum_{u v \notin E(G)} \frac{1}{\sqrt{r s(u)+r s(v)}} .
$$

The reciprocal transmission atom-bond connectivity co-index of a graph $G$ is denoted by $\overline{R T}_{A B C}(G)$ and it is defined by

$$
\overline{R T}_{A B C}(G)=\sum_{u v \notin E(G)} \sqrt{\frac{r s(u)+r s(v)-2}{r s(u) r s(v)}} .
$$

The reciprocal transmission augmented Zagreb co-index of a graph $G$ is denoted by $\overline{R T}_{A Z}(G)$ and it is defined by

$$
\overline{R T}_{A Z}(G)=\sum_{u v \notin E(G)}\left[\frac{r s(u) r s(v)}{r s(u)+r s(v)-2}\right]^{3} .
$$

We have already obtained explicit formulae for transmission and reciprocal transmission based topological coindices in terms of order and size. In the following, we obtain bounds for the above defined transmission and reciprocal transmission based topological co-indices. 


\section{Bounds for transmission and reciprocal transmission based topological co-indices}

Now we obtain inequalities for transmission and reciprocal transmission based topological coindices:

Theorem 1. Let $G$ be a connected graph with $n$ vertices and let $D=\operatorname{diam}(G)$. Then

$$
\begin{gathered}
\sum_{u v \notin E(G)} \frac{1}{\sqrt{2 D(n-1)-(D-1)(d(u)+d(v))}} \leq \bar{T}_{S C}(G) \\
\leq \sum_{u v \notin E(G)} \frac{1}{\sqrt{4 n-4-(d(u)+d(v))}} .
\end{gathered}
$$

Equality holds on both sides if and only if $\operatorname{diam}(G) \leq 2$.

Proof. For any vertex $u$ of $G$, there are $d(u)$ vertices which are at distance 1 from $u$. Further, the distance between $u$ and remaining $n-1-d(u)$ vertices is at least 2 and at most $D$. Therefore

$$
\sigma(u) \leq d(u)+D(n-1-d(u))=D(n-1)-(D-1) d(u)
$$

and

$$
\sigma(u) \geq d(u)+2(n-1-d(u))=2 n-2-d(u)
$$

with equality in both cases if and only if $D=2$. Therefore,

$$
4 n-4-(d(u)+d(v)) \leq \sigma(u)+\sigma(v) \leq 2 D(n-1)-(D-1)(d(u)+d(v)) .
$$

Hence

$$
\bar{T}_{S C}(G)=\sum_{u v \notin E(G)} \frac{1}{\sqrt{\sigma(u)+\sigma(v)}} \geq \sum_{u v \notin E(G)} \frac{1}{\sqrt{2 D(n-1)-(D-1)(d(u)+d(v))}}
$$

and

$$
\bar{T}_{S C}(G)=\sum_{u v \notin E(G)} \frac{1}{\sqrt{\sigma(u)+\sigma(v)}} \leq \sum_{u v \notin E(G)} \frac{1}{\sqrt{4 n-4-(d(u)+d(v))}} .
$$

Equality holds in both cases if and only if $D=2$.

Theorems 2, 3, 4 and 5 can be proved analogously to Theorem 1 .

Theorem 2. Let $G$ be a connected graph with $n$ vertices and let $D=\operatorname{diam}(G)$. Then

$$
\begin{gathered}
\sum_{u v \notin E(G)} \sqrt{\frac{2 D(n-1)-(D-1)(d(u)+d(v))-2}{(D(n-1))^{2}-D(n-1)(D-1)\left(d(u)+d(v)-(D-1)^{2} d(u) d(v)\right.}} \leq \bar{T}_{A B C}(G) \\
\leq \sum_{u v \notin E(G)} \sqrt{\frac{4 n-6-(d(u)+d(v))}{4 n^{2}-8 n-4+(2-2 n)(d(u)+d(v))+d(u) d(v)}}
\end{gathered}
$$


Theorem 3. Let $G$ be a connected graph with $n$ vertices and let $D=\operatorname{diam}(G)$. Then

$$
\begin{gathered}
\sum_{u v \notin E(G)} \frac{2 D(n-1)-(D-1)(d(u)+d(v))}{2 \sqrt{(D(n-1))^{2}-D(n-1)(D-1)(d(u)+d(v))+(D-1)^{2} d(u) d(v)}} \leq \bar{T}_{A G}(G) \\
\leq \sum_{u v \notin E(G)} \frac{4 n-4-(d(u)+d(v))}{2 \sqrt{(2 n-2-d(u))(2 n-2-d(v))}} .
\end{gathered}
$$

Theorem 4. Let $G$ be a connected graph with $n$ vertices and let $D=\operatorname{diam}(G)$. Then

$$
\begin{gathered}
\sum_{u v \notin E(G)}\left[\frac{(D(n-1))^{2}-D(n-1)(D-1)(d(u)+d(v))-(D-1)^{2} d(u) d(v)}{2 D(n-1)+(D-1)(d(u)+d(v))-2}\right]^{3} \leq \bar{T}_{A Z}(G) \\
\leq \sum_{u v \notin E(G)}\left[\frac{4 n^{2}-8 n-4+(2-2 n)(d(u)+d(v))+d(u) d(v)}{4 n-6-(d(u)+d(v))}\right]
\end{gathered}
$$

Theorem 5. Let $G$ be a connected graph with $n$ vertices and let $D=\operatorname{diam}(G)$. Then,

$$
\begin{gathered}
\sum_{u v \notin E(G)} \frac{2 \sqrt{(D(n-1)-(D-1) d(u))(D(n-1)-(D-1) d(v))}}{2 D(n-1)-(D-1)(d(u)+d(v))} \leq \bar{T}_{G A} \\
\sum_{u v \notin E(G)} \frac{2 \sqrt{(2 n-2-d(u))(2 n-2-d(v))}}{4 n-4-(d(u)+d(v))} .
\end{gathered}
$$

Theorem 6. Let $G$ be a connected graph with $n$ vertices and let $\operatorname{diam}(G)=D$. Then,

$$
\begin{gathered}
\sum_{u v \notin E(G)} \frac{1}{\sqrt{(n-1)+\frac{1}{2}(d(u)+d(v))}} \leq \overline{R T}_{S C}(G) \leq \\
\sum_{u v \notin E(G)} \frac{1}{\sqrt{\frac{2(n-1)}{D}+\left(1-\frac{1}{D}\right)(d(u)+d(v))}} .
\end{gathered}
$$

Proof. Lower bound: For any vertex $u$ of $G$ there are $d(u)$ vertices which are at distance 1 from $u$ and remaining $n-1-d(u)$ vertices are at distance at least 2 . Therefore $r s(u) \leq \frac{1}{2}(n-1+d(u))$ and $r s(u)+r s(v) \leq(n-1)+\frac{1}{2}(d(u)+d(v))$. We have

$$
\overline{R T}_{S C}(G)=\sum_{u v \notin E(G)} \frac{1}{\sqrt{r s(u)+r s(v)}}
$$




$$
\geq \sum_{u v \notin E(G)} \frac{1}{\sqrt{(n-1)+\frac{1}{2}(d(u)+d(v))}} .
$$

Upper bound: For any vertex $u$ of $G$ there are $d(u)$ vertices which are at distance 1 from $u$ and remaining $n-1-d(u)$ vertices are at distance at most $D$. Therefore $\operatorname{rs}(u) \geq$ $\frac{1}{D}(n-1)+\left(1-\frac{1}{D}\right) d(u)$ and $r s(u)+r s(v) \geq \frac{2(n-1)}{D}+\left(1-\frac{1}{D}\right)(d(u)+d(v))$. We have

$$
\overline{R T}_{S C}(G) \leq \sum_{u v \notin E(G)} \frac{1}{\sqrt{\frac{2(n-1)}{D}+\left(1-\frac{1}{D}\right)(d(u)+d(v))}} .
$$

Theorems 7, 8, 9 and 10 can be proved analogously to Theorem 6 :

Theorem 7. Let $G$ be a connected graph with $n$ vertices and let $\operatorname{diam}(G)=D$. Then

$$
\begin{gathered}
\sum_{u v \notin E(G)} \sqrt{\frac{2(2(n-1)+d(u)+d(v)-4)}{(n-1)^{2}+(n-1)(d(u)+d(v))+d(u) d(v)}} \leq \overline{R T}_{A B C}(G) \leq \\
\sum_{u v \notin E(G)} \sqrt{\frac{\frac{2(n-1)}{D}+\left(1-\frac{1}{D}\right)(d(u)+d(v))-2}{\left(\frac{1}{D}(n-1)\right)^{2}+\left(1-\frac{1}{D}\right) d(u) d(v)+\frac{(n-1)}{D}\left(1-\frac{1}{D}\right)(d(u)+d(v))}} .
\end{gathered}
$$

Theorem 8. Let $G$ be a connected graph with $n$ vertices and let $\operatorname{diam}(G)=D$. Then

$$
\begin{gathered}
\sum_{u v \notin E(G)}\left[\frac{(n-1)^{2}+(n-1)(d(u)+d(v))+d(u) d(v)}{2(2(n-1)+d(u)+d(v)-4)}\right]^{3} \leq \overline{R T}_{A Z}(G) \leq \\
\sum_{u v \notin E(G)}\left[\frac{\left(\frac{1}{D}(n-1)\right)^{2}+\left(1-\frac{1}{D}\right) d(u) d(v)+\frac{n-1}{D}\left(1-\frac{1}{D}\right)(d(u)+d(v))}{\frac{2(n-1)}{D}+\left(1-\frac{1}{D}\right)(d(u)+d(v))-2}\right]^{3} .
\end{gathered}
$$

Theorem 9. Let $G$ be a connected graph with $n$ vertices and let $\operatorname{diam}(G)=D$. Then

$$
\begin{gathered}
\sum_{u v \notin E(G)} \frac{2(n-1)+(d(u)+d(v))}{2 \sqrt{(n-1+d(u))(n-1+d(v))}} \leq \overline{R T}_{A G}(G) \leq \\
\sum_{u v \notin E(G)} \frac{\frac{2(n-1)}{D}+\left(1-\frac{1}{D}\right)(d(u)+d(v))}{2 \sqrt{\left(\frac{1}{D}(n-1)\right)^{2}+\frac{1}{D}(n-1)\left(1-\frac{1}{D}\right)(d(u)+d(v))+\left(1-\frac{1}{D}\right)^{2} d(u) d(v)}} .
\end{gathered}
$$


Theorem 10. Let $G$ be a connected graph with $n$ vertices and let $\operatorname{diam}(G)=D$. Then

$$
\begin{gathered}
\sum_{u v \notin E(G)} \frac{2 \sqrt{(n-1+d(u))(n-1+d(v))}}{2(n-1)+(d(u)+d(v))} \leq \overline{R T}_{G A}(G) \leq \\
\sum_{u v \notin E(G)} \frac{2 \sqrt{\left(\frac{1}{D}(n-1)\right)^{2}+\frac{1}{D}(n-1)\left(1-\frac{1}{D}\right)(d(u)+d(v))+\left(1-\frac{1}{D}\right)^{2} d(u) d(v)}}{\frac{2(n-1)}{D}+\left(1-\frac{1}{D}\right)(d(u)+d(v))} .
\end{gathered}
$$

\section{Transmission and reciprocal transmission based topological co-indices of some graphs}

For any vertex $u$ of a complete graph $K_{n}$, we have $\sigma(u)=n-1$. Hence we get the following result:

Proposition 1. For a complete graph $K_{n}$ on $n$ vertices,

$$
\begin{array}{cccc}
\bar{T}_{S C}\left(K_{n}\right)=0, & \bar{T}_{A B C}\left(K_{n}\right)=0, & \bar{T}_{A G}\left(K_{n}\right)=0, & \bar{T}_{A Z}\left(K_{n}\right)=0, \\
\bar{T}_{G A}\left(K_{n}\right)=0, & \overline{R T}_{S C}\left(K_{n}\right)=0, & \overline{R T}_{A B C}\left(K_{n}\right)=0, & \overline{R T}_{A G}\left(K_{n}\right)=0, \\
\overline{R T}_{A Z}\left(K_{n}\right)=0, & \overline{R T}_{G A}\left(K_{n}\right)=0 . &
\end{array}
$$

The vertex set of a complete bipartite graph $K_{p, q}$ can be partitioned into two sets $V_{1}$ and $V_{2}$ such that every edge of $K_{p, q}$ has one end in $V_{1}$ and other end in $V_{2}$, where $\left|V_{1}\right|=p$ and $\left|V_{2}\right|=q$. If the vertex $u \in V_{1}$ and $v \in V_{2}$, then $d(u)=p$ and $d(v)=q$. Recall that the graph $K_{p, q}$ has $n=p+q$ vertices and $m=p q$ edges. Also $\operatorname{diam}\left(K_{p, q}\right) \leq 2$. Therefore by the equality part of Theorems $1,2,3$ and 4 , we get the following result:

Proposition 2. For a complete bipartite graph $K_{p, q}$, we have

$$
\begin{aligned}
\bar{T}_{S C}\left(K_{p, q}\right) & =\left(\left(\begin{array}{c}
p+q \\
2
\end{array}\right)-p q\right) \frac{1}{\sqrt{3(p+q)-4}}, \\
\bar{T}_{A B C}\left(K_{p, q}\right) & =\left(\left(\begin{array}{c}
p+q \\
2
\end{array}\right)-p q\right) \sqrt{\frac{3(p+q)-6}{(p+2(q-1))(q+2(p-1))}} \\
\bar{T}_{A G}\left(K_{p, q}\right) & =\left(\left(\begin{array}{c}
p+q \\
2
\end{array}\right)-p q\right)\left(\frac{3(p+q)-4}{2 \sqrt{(p+2(q-1))(q+2(p-1))}}\right) \\
\bar{T}_{G A}\left(K_{p, q}\right) & =\left(\left(\begin{array}{c}
p+q \\
2
\end{array}\right)-p q\right)\left(\frac{2 \sqrt{(p+2(q-1))(q+2(p-1))}}{3(p+q)-4}\right) \\
\bar{T}_{A Z}\left(K_{p, q}\right) & =\left(\left(\begin{array}{c}
p+q \\
2
\end{array}\right)-p q\right)\left(\frac{(p+2(q-1))(q+2(p-1))}{3(p+q)-6}\right)^{3} \\
\overline{R T}_{S C}\left(K_{p, q}\right) & =\left(\left(\begin{array}{c}
p+q \\
2
\end{array}\right)-p q\right)\left(\frac{1}{\sqrt{\frac{3}{2}(p+q)-1}}\right)
\end{aligned}
$$




$$
\begin{aligned}
\overline{R T}_{A B C}\left(K_{p, q}\right) & =\left(\left(\begin{array}{c}
p+q \\
2
\end{array}\right)-p q\right) \sqrt{\frac{\frac{3}{2}(p+q)-3}{(p+2(q-1))(q+2(p-1))}} \\
\bar{T}_{A G}\left(K_{p, q}\right) & =\left(\left(\begin{array}{c}
p+q \\
2
\end{array}\right)-p q\right)\left(\frac{\frac{3}{2}(p+q)-1}{2 \sqrt{(p+2(q-1))(q+2(p-1))}}\right) \\
\overline{R T}_{G A}\left(K_{p, q}\right) & =\left(\left(\begin{array}{c}
p+q \\
2
\end{array}\right)-p q\right)\left(\frac{2 \sqrt{(p+2(q-1))(q+2(p-1))}}{\frac{3}{2}(p+q)-1}\right) \\
\overline{R T}_{A Z}\left(K_{p, q}\right) & =\left(\left(\begin{array}{c}
p+q \\
2
\end{array}\right)-p q\right)\left(\frac{(p+2(q-1)(q+2(p-1))}{\frac{3}{2}(p+q)-3}\right)^{3} .
\end{aligned}
$$

For any vertex $u$ of a cycle $C_{n}$ on $n \geq 3$ vertices, we have

$$
\sigma(u)= \begin{cases}2\left[1+2+\cdots+\frac{n-1}{2}\right]+\frac{n}{2}=\frac{n^{2}}{4}, & \text { if } \quad n \text { is even } \\ 2\left[1+2+\cdots+\frac{n-1}{2}\right]=\frac{n^{2}-1}{4}, & \text { if } \quad n \text { is odd }\end{cases}
$$

and

$$
r s(u)= \begin{cases}2 \sum_{i=1}^{\frac{n-2}{2}} \frac{1}{i}+\frac{2}{n}, & \text { if } n \text { is even } \\ 2 \sum_{i=1}^{\frac{n-1}{2}} \frac{1}{i}, & \text { if } n \text { is odd. }\end{cases}
$$

Proposition 3. For a cycle $C_{n}$ on $n \geq 3$ vertices, we have

$$
\begin{aligned}
& \bar{T}_{S C}\left(C_{n}\right)= \begin{cases}\left(\left(\begin{array}{l}
n \\
2
\end{array}\right)-n\right) \sqrt{\frac{2}{n^{2}}}, & \text { if } n \text { is even } \\
\left(\left(\begin{array}{l}
n \\
2
\end{array}\right)-n\right) \sqrt{\frac{2}{n^{2}-1}}, & \text { if } n \text { is odd }\end{cases} \\
& \bar{T}_{A B C}\left(C_{n}\right)=\left\{\begin{array}{lll}
\left(\left(\begin{array}{l}
n \\
2
\end{array}\right)-n\right) \sqrt{\frac{8\left(n^{2}-4\right)}{n^{4}}}, & \text { if } & n \text { is even } \\
\left(\left(\begin{array}{l}
n \\
2
\end{array}\right)-n\right) \sqrt{\frac{8\left(n^{2}-5\right)}{\left(n^{2}-1\right)^{2}}}, & \text { if } n \text { is odd }
\end{array}\right.
\end{aligned}
$$

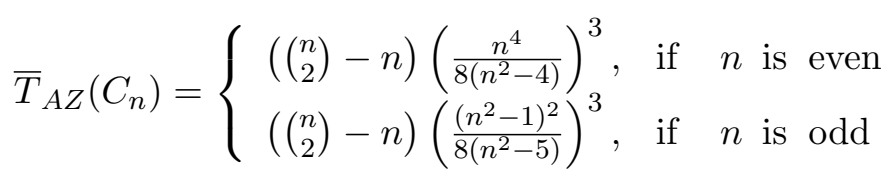

$$
\begin{aligned}
& \bar{T}_{A G}\left(C_{n}\right)=\left(\left(\begin{array}{l}
n \\
2
\end{array}\right)-n\right) \text { for any value of } n \text {. } \\
& \bar{T}_{G A}\left(C_{n}\right)=\left(\left(\begin{array}{l}
n \\
2
\end{array}\right)-n\right) \text { for any value of } n \text {. }
\end{aligned}
$$


H. S. Ramane, S. Y. Talwar, I. N. Cangul / Eur. J. Pure Appl. Math, 13 (5) (2020), 1057-1071 1067

$$
\begin{aligned}
& \overline{R T}_{S C}\left(C_{n}\right)= \begin{cases}\left(\left(\begin{array}{l}
n \\
2
\end{array}\right)-n\right) \frac{1}{2 \sqrt{\sum_{i=1}^{\frac{n-2}{2}} \frac{1}{i}+\frac{1}{n}}}, & \text { if } n \text { is even } \\
\left(\left(\begin{array}{l}
n \\
2
\end{array}\right)-n\right) \frac{(1)}{2 \sqrt{\sum_{i=1}^{\frac{n-1}{2}} \frac{1}{i}}}, & \text { if } n \text { is odd }\end{cases}
\end{aligned}
$$

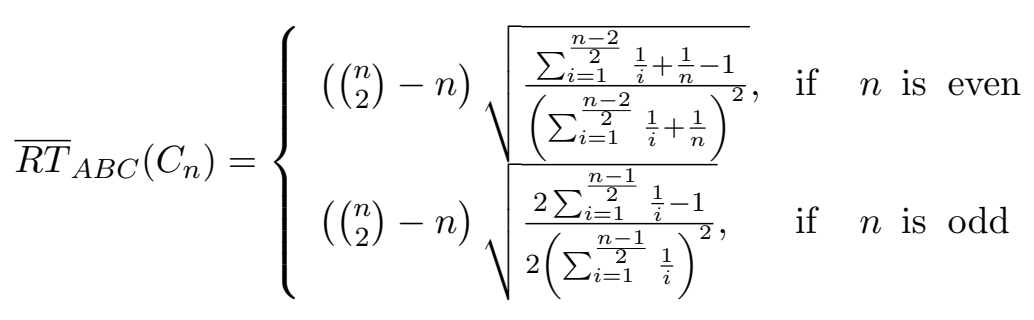

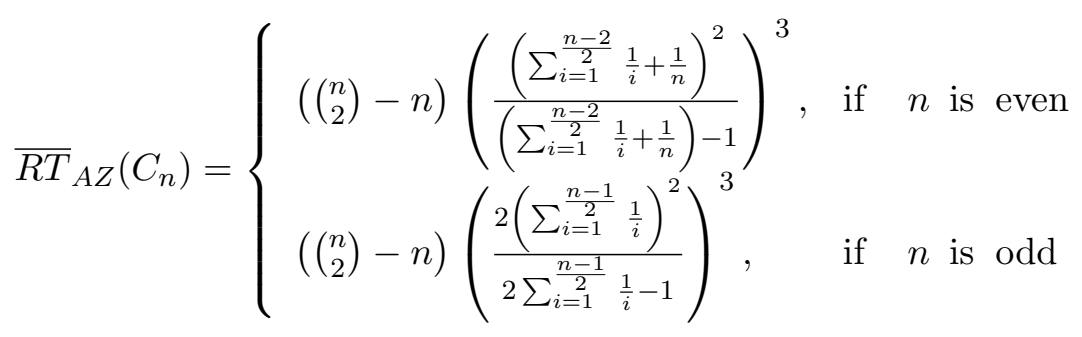

$$
\begin{aligned}
& \overline{R T}_{A G}\left(C_{n}\right)=\left(\left(\begin{array}{l}
n \\
2
\end{array}\right)-n\right) \text { for any value of } n \\
& \overline{R T}_{G A}\left(C_{n}\right)=\left(\left(\begin{array}{l}
n \\
2
\end{array}\right)-n\right) \text { for any value of } n
\end{aligned}
$$

A wheel $W_{n+1}$ is a graph obtained from the cycle $C_{n}, n \geq 3$, by adding a new vertex and making it adjacent to all the vertices of $C_{n}$. The degree of a central vertex of $W_{n+1}$ is $n$ and the degree of all other vertices is 3 . Hence

Proposition 4. For a wheel $W_{n+1}, n \geq 3$,

$$
\begin{gathered}
\bar{T}_{S C}\left(W_{n+1}\right)=\left(\left(\begin{array}{c}
n+1 \\
2
\end{array}\right)-2 n\right) \frac{1}{\sqrt{4 n-6}}, \\
\bar{T}_{A B C}\left(W_{n+1}\right)=\left(\left(\begin{array}{c}
n+1 \\
2
\end{array}\right)-2 n\right) \sqrt{\frac{4 n-8}{4 n^{2}-12 n+9}}, \\
\bar{T}_{A Z}\left(W_{n+1}\right)=\left(\left(\begin{array}{c}
n+1 \\
2
\end{array}\right)-2 n\right)\left(\frac{4 n^{2}-12 n+9}{4 n-8}\right)^{3},
\end{gathered}
$$


H. S. Ramane, S. Y. Talwar, I. N. Cangul / Eur. J. Pure Appl. Math, 13 (5) (2020), 1057-1071 1068

$$
\begin{aligned}
& \bar{T}_{G A}\left(W_{n+1}\right)=\left(\left(\begin{array}{c}
n+1 \\
2
\end{array}\right)-2 n\right)\left(\frac{2 \sqrt{4 n^{2}-12 n+9}}{4 n-6}\right), \\
& \bar{T}_{A G}\left(W_{n+1}\right)=\left(\left(\begin{array}{c}
n+1 \\
2
\end{array}\right)-2 n\right)\left(\frac{4 n-6}{2 \sqrt{4 n^{2}-12 n+9}}\right) \\
& \overline{R T}_{A G}\left(W_{n+1}\right)=\left(\left(\begin{array}{c}
n+1 \\
2
\end{array}\right)-2 n\right)\left(\frac{n+3}{2 \sqrt{\left(3+\frac{1}{2}(n-3)\right)^{2}}}\right), \\
& \overline{R T}_{G A}\left(W_{n+1}\right)=\left(\left(\begin{array}{c}
n+1 \\
2
\end{array}\right)-2 n\right)\left(\frac{2 \sqrt{\left(3+\frac{1}{2}(n-3)\right)^{2}}}{n+3}\right), \\
& \overline{R T}_{S C}\left(W_{n+1}\right)=\left(\left(\begin{array}{c}
n+1 \\
2
\end{array}\right)-2 n\right) \frac{1}{\sqrt{n+3}}, \\
& \overline{R T}_{A B C}\left(W_{n+1}\right)=\left(\left(\begin{array}{c}
n+1 \\
2
\end{array}\right)-2 n\right) \sqrt{\frac{n+1}{\left(3+\frac{1}{2}(n-3)\right)^{2}}}, \\
& \overline{R T}_{A Z}\left(W_{n+1}\right)=\left(\left(\begin{array}{c}
n+1 \\
2
\end{array}\right)-2 n\right)\left(\frac{\left(3+\frac{1}{2}(n-3)^{2}\right)}{n+1}\right)^{3}
\end{aligned}
$$

A friendship graph (or Dutch windmill graph) $F_{n}, n \geq 2$, is a graph that can be constructed by coalescence $n$ copies of the cycle $C_{3}$ of length 3 with a common vertex. It has $2 n+1$ vertices and $3 n$ edges. The degree of a coalescence vertex of $F_{n}$ is $2 n$ and the degree of all other vertices is 2 .

Proposition 5. For a friendship graph $F_{n}, n \geq 2$,

$$
\begin{gathered}
\bar{T}_{S C}\left(F_{n}\right)=\left(\left(\begin{array}{c}
2 n+1 \\
2
\end{array}\right)-3 n\right) \frac{1}{2 \sqrt{2 n-1}}, \\
\bar{T}_{A B C}\left(F_{n}\right)=\left(\left(\begin{array}{c}
2 n+1 \\
2
\end{array}\right)-3 n\right) \sqrt{\frac{4 n-3}{8 n^{2}-8 n+2}} \\
\bar{T}_{A Z}\left(F_{n}\right)=\left(\left(\begin{array}{c}
2 n+1 \\
2
\end{array}\right)-3 n\right)\left(\frac{8 n^{2}-8 n+2}{4 n-3}\right)^{3},
\end{gathered}
$$




$$
\begin{gathered}
\bar{T}_{G A}\left(F_{n}\right)=\left(\left(\begin{array}{c}
2 n+1 \\
2
\end{array}\right)-3 n\right)\left(\frac{\sqrt{4 n^{2}-4 n+1}}{2 n-1}\right), \\
\bar{T}_{A G}\left(F_{n}\right)=\left(\left(\begin{array}{c}
2 n+1 \\
2
\end{array}\right)-3 n\right)\left(\frac{2 n-1}{\sqrt{4 n^{2}-4 n+1}}\right), \\
\overline{R T}_{A G}\left(F_{n}\right)=\left(\begin{array}{c}
2 n+1 \\
2
\end{array}\right)-3 n \\
\overline{R T}_{G A}\left(F_{n}\right)=\left(\begin{array}{c}
2 n+1 \\
2
\end{array}\right)-3 n, \\
\overline{R T}_{S C}\left(F_{n}\right)=\left(\left(\begin{array}{c}
2 n+1 \\
2
\end{array}\right)-3 n\right) \frac{1}{\sqrt{2(n+1)}}, \\
\overline{R T}_{A B C}\left(F_{n}\right)=\left(\left(\begin{array}{c}
2 n+1 \\
2
\end{array}\right)-3 n\right) \frac{\sqrt{2 n}}{n+1}, \\
\overline{R T}_{A Z}\left(F_{n}\right)=\left(\left(\begin{array}{c}
2 n+1 \\
2
\end{array}\right)-3 n\right)\left(\frac{(n+1)^{2}}{2 n}\right)^{3} .
\end{gathered}
$$

Acknowledgements The first author HSR is thankful to University Grants Commission (UGC), New Delhi, for the support through grant under UGC-SAP DRS-III, 2016-2021: F.510/3/DRS-III /2016 (SAP-I). The second author SYT is thankful to Ministry of Tribal Affairs, Govt. of India, New Delhi for awarding National Fellowship for Higher Education No. 2017 18-NFST-KAR-01182.

\section{References}

[1] B Basvanagoud, V R Desai, and I N Cangul. Four new tensor products of graphs and their zagreb indices and coindices. Electronic Journal of Mathematical Analysis and Applications, 8(1):209-219, 2020.

[2] A R Bindusree, I N Cangul, V Lokesha, and A S Cevik. Zagreb Polynomials of three Graph Operators. Filomat, 30(7):1979-1986, 2016.

[3] K C Das, N Akgunes, M Togan, A Yurttas, I N Cangul, and A S Cevik. On the first Zagreb index and multiplicative Zagreb coindices of graphs. Analele Stiintifice ale Universitatii Ovidius Constanta, 24(1):153-176, 2016. 
[4] K C Das, A Yurttas, M Togan, I N Cangul, and A S Cevik. The multiplicative zagreb indices of graph operations. Journal of Inequalities and Applications., 90:1-14, 2013.

[5] B Furtula and I Gutman. A forgotten topological index. J Math Chem, 53(4):11841190, 2015.

[6] I Gutman. Degree-based topological indices. Analele Stiintifice ale Universitatii Ovidius Constanta, 86:351-361, 2013.

[7] I Gutman and K C Das. The first Zagreb index 30 years after. MATCH Commu. Math. Comput. Chem., 50:83-92, 2004.

[8] I Gutman and N Trinajstić. Graph theory and molecular orbitals, Total $\pi$-electron energy of alternant hydrocarbons. Chem. Phys. Lett., 17:535-538, 1972.

[9] F Harary. Status and contrastatus. Sociometry, 22(1):23-43, 1959.

[10] F Harary. Graph Theory. Narosa Publishing House, New Delhi, 1999.

[11] K P Narayankar and D Selvan. Geometric arithmetic status index of graphs. Int. J. Math. Arch., 8:230-233, 2017.

[12] S Nikolić, G Kovačević, A Miličević, and N Trinajstić. The Zagreb indices 30 years after. Croat. Chem. Acta, 76:113-124, 2003.

[13] D Plavi, S Nikoli, N Trinajsti, and Z Mihali. On the Harary Index for the Characterization of Chemical Graphs. J. Math. Chem., 12:235-250, 1993.

[14] H S Ramane and S Y Talwar. Reciprocal transmission based topological indices of graphs and its applications in chemistry. Preprint.

[15] H S Ramane and S Y Talwar. Transmission based topological indices of graphs and its regression analysis with some molecular properties. Preprint.

[16] H S Ramane and A S Yalnaik. Status connectivity indices of graphs and its applications to the boiling point of benzenoid hydrocarbons. J. Appl. Math. Comput., 55:609-627, 2017.

[17] P S Ranjini, V Lokesha, and I N Cangul. On the Zagreb Indices of the Line Graphs of the Subdivision Graphs. Applied Mathematics and Computation, 218(3):699-702, 2011.

[18] P Sarkar, N De, I N Cangul, and A Pal. The (a,b)-Zagreb index of some derived networks. Journal of Taibah University for Science, 13(1):79-86, 2019.

[19] R Sharafdini and T Reti. On the transmission-based graph topological indices. Kragujevac J. Math., 44:41-63, 2020. 
[20] M Togan, A Yurttas, and I N Cangul. All versions of Zagreb indices and coindices of subdivision graphs of certain graph types. Advanced Studies in Contemporary Mathematics, 26(1):227-236, 2016.

[21] M Togan, A Yurttas, and I N Cangul. Zagreb and multiplicative Zagreb indices of r-subdivision graphs of double graphs. Scientia Magna, 12(1):115-119, 2017.

[22] M Togan, A Yurttas, and I N Cangul. Inverse problem for the first entire Zagreb index. Advanced Studies in Contemporary Mathematics, 29(2):161-169, 2019.

[23] M Togan, A Yurttas, A S Cevik, and I N Cangul. Effect of Edge Deletion and Addition on Zagreb Indices of Graphs. In: Ta, K., Baleanu, D., Machado, J. (eds), Mathematical Methods in Engineering, Theoretical Aspects. Nonlinear Systems and Complexity, 23:191-201, 2019.

[24] M Togan, A Yurttas, A S Cevik, and I N Cangul. Indices and Multiplicative Zagreb Indices of Double Graphs of Subdivision Graphs. Turkic World of Mathematical Society, Journal of Applied and Engineering Mathematics, 9(2):404-412, 2019.

[25] H Wiener. Structural determination of paraffin boiling points. J. Am. Chem. Soc., 69:17-20, 1947.

[26] A Yurttas, M Togan, and I N Cangul. Zagreb indices and multiplicative Zagreb indices of subdivision graphs of double graphs. Advanced Studies in Contemporary Mathematics, 26(3):407-416, 2016.

[27] A Yurttas, M Togan, and I N Cangul. Zagreb Indices of Graphs with Added Edges. Proceedings of the Jangjeon Mathematical Society, 21(3):385-392, 2018.

[28] A Yurttas, M Togan, V Lokesha, I N Cangul, and I Gutman. Inverse problem for Zagreb indices. Journal of Mathematical Chemistry, 57:609-615, 2019. 